\title{
Semianalytic model of electron pulse propagation: Magnetic lenses and rf pulse compression cavities
}

\author{
Joel A. Berger ${ }^{\mathrm{a})}$ and W. Andreas Schroeder \\ Department of Physics, University of Illinois at Chicago, 845 W. Taylor (M/C 273), Chicago, \\ Illinois 60607 USA
}

(Received 22 December 2009; accepted 7 October 2010; published online 23 December 2010)

\begin{abstract}
The analytical Gaussian electron pulse propagation model of Michalik and Sipe [J. Appl. Phys. 99, 054908 (2006)] is extended to include the action of external forces on the pulse. The resultant ability to simulate efficiently the effect of electron optical elements (e.g., magnetic lenses and radio-frequency cavities) allows for the rapid assessment of electron pulse delivery systems in time-resolved ultrafast electron diffraction and microscopy experiments. (C) 2010 American Institute of Physics. [doi:10.1063/1.3512847]
\end{abstract}

\section{INTRODUCTION}

The development of laboratory-scale instrumentation for the direct visualization of fundamental ultrafast (i.e., subnanosecond) events with nanoscale (even atomic) spatial resolution is a current active area of research. ${ }^{2}$ In many areas of physics, chemistry and materials science a space-time resolution in the sub-1 $\mathrm{nm}$ ps range is ideally required to allow for the observation of the fastest structural events. A promising approach is to employ ultrashort pulses of electrons generated by a laser-driven $10-100 \mathrm{keV}$ photoelectron gun to probe the dynamics of the specimen that has been perturbed by a synchronized laser pulse. ${ }^{2}$ Ultrafast electron diffraction (UED) or femtosecond electron diffraction (FED) (Refs. $3-5)$ is an example of this type of technique and has been used successfully to elucidate atomic-scale dynamics in melting, ${ }^{5-7}$ chemical bond breaking, ${ }^{8}$ etc. Direct timeresolved imaging of similar events can be accomplished using transmission electron microscopy (TEM). To date, such dynamic TEMs (DTEMs) (Refs. 9 and 10) have been developed for both single-shot imaging with $\sim 10^{5} \mathrm{~nm}$ ps spacetime resolution (e.g., $30 \mathrm{~ns}$ electron pulses and 5-10 nm spatial resolution ${ }^{11,12}$ ) and $\sim 1 \mathrm{~nm}$ ps space-time resolution $\left(\sim 100\right.$ fs with a few nanometer resolution $\left.{ }^{13}\right)$ in the multishot data acquisition mode of operation. Both of these instruments employ retrofitted standard electron microscope columns and are limited in performance mainly by space-charge effects.

Improvements in the performance of DTEMs, especially ultrafast electron microscopes (UEMs), and UED systems will likely require the implementation of techniques that offset or compensate for deleterious space-charge effects. ${ }^{14,15}$ Such performance optimization efforts will require efficient models of electron pulse propagation dynamics that include the ability to simulate the action of electron optical elements (including the photoelectron gun itself) on the pulse. Indeed second generation UED instruments are now beginning to employ both magnetic lenses and radio-frequency (rf) cavities $^{16,17}$ to optimize the electron pulse delivery to the specimen; that is, compensate for global space-charge effects

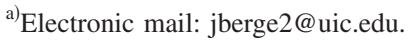

in both the transverse and longitudinal pulse dimensions, respectively. Similarly, future UEM column designs are expected to use rf pulse compression cavities while circumventing high charge density beam cross-overs (i.e., beam foci).

In this paper, we present an extension of the work of Michalik and Sipe, ${ }^{1}$ describing electron bunch propagation in an analytic mean-field self-similar Gaussian (AG) pulse treatment, to include the influence of linear external forces due to electron optical elements. The resulting computationally efficient propagation analysis can then be used to model, and hence design, UEM columns and UED systems in a straightforward manner. For completeness, in Sec. II we present, in MKS units, the essential features of the AG model before discussing the basic operational parameters for a DTEM, equipped with a laser-driven photoelectron gun, in Sec. III. In Sec. IV, we outline the extension of the Michalik and Sipe AG model to include external forces, for which examples of magnetic lenses and rf compression cavities are then shown in Sec. V. Finally, before concluding, we present in Sec. VI an exemplar combination of electron optical elements for the delivery of a space-time optimized ultrashort electron pulse to the vicinity of the specimen location.

We emphasize that the presented extension to the AG model to include external forces acting on the electron pulse is valid only within the limits of the analytical method itself; in particular, its mean internal space-charge field and selfsimilar Gaussian approximations. ${ }^{1}$ As a result, the extension reflects a first-order (i.e., linear force) analysis of the effects of electron optics upon electron pulse propagation. Nonetheless, for free-space propagation, the AG model of charge bunch dynamics has already been shown to be very consistent with full Monte Carlo (i.e., particle tracking) simulations for a wide variety of electron pulse shapes, ${ }^{1,18}$ including the uniform ellipsoid. ${ }^{19}$ This successful benchmarking is due primarily to the versatility of the AG model which results from its use of transverse and longitudinal pulse position and momentum variances. Consequently, the AG approach is applicable to both the single electron per pulse limit, ${ }^{15}$ where momentum variances determine the pulse evolution and the model is exact (obeying Gaussian optics), and the high 


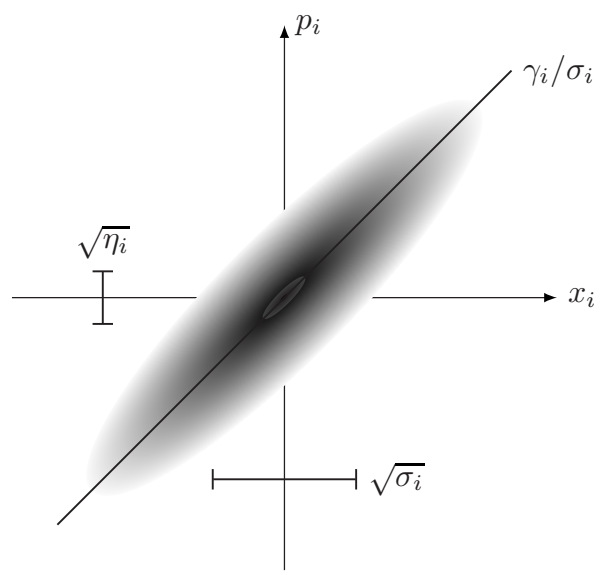

FIG. 1. A schematic depiction showing the AG model parameters in the representation of the usual position-momentum phase-space plots: $\sqrt{\sigma_{i}}$ and $\sqrt{\eta_{i}}$ are the variances of the $i$ direction spatial and momentum distributions, respectively, and the slope, given by $\gamma_{i} / \sigma_{i}$, is related to the (linear) momentum chirp of the pulse.

charge density limit in which space-charge effects dominate. ${ }^{5,19,20}$ It is this versatility combined with its computational efficiency that makes the presented extended AG model particularly suitable for rapid initial assessments of pulsed electron microscope column designs and electron pulse delivery systems in UED experiments. Verification of the validity (and determination of the limits) of the extended AG model will, of course, require future comparison with both experiment and more complete simulations of electron pulse propagation dynamics (e.g., full particle tracking models) that include nonlinear forces, for both the intrapulse space-charge field and the description of aberrations in electron optics.

We also note that our analyses are performed in the nonrelativistic limit, which is a reasonable approximation for the typical $20-200 \mathrm{keV}$ electron energies employed in UED and electron microscopy. Extension to include relativistic effects is straightforward using standard transformations between the laboratory and electron pulse reference frames.

\section{ELECTRON PULSE PROPAGATION MODEL}

In their mean-field analytical formalism of electron pulse propagation, Michalik and Sipe ${ }^{1}$ characterize the electron pulse in position and momentum space (in the pulse frame) by a normalized Gaussian distribution function:

$$
\begin{aligned}
f\left(x, y, z, p_{x}, p_{y}, p_{z}\right) & =\frac{N}{(2 \pi)^{3} \sigma_{T} \eta_{T} \sqrt{\sigma_{z} \eta_{z}}} \exp \left[-\left(\frac{x^{2}}{2 \sigma_{T}}+\frac{y^{2}}{2 \sigma_{T}}+\frac{z^{2}}{2 \sigma_{z}}\right.\right. \\
& \left.\left.+\frac{\left(p_{x}-\frac{\gamma_{T} x}{\sigma_{T}}\right)^{2}}{2 \eta_{T}}+\frac{\left(p_{y}-\frac{\gamma_{T} y}{\sigma_{T}}\right)^{2}}{2 \eta_{T}}+\frac{\left(p_{z}-\frac{\gamma_{z} z}{\sigma_{z}}\right)^{2}}{2 \eta_{z}}\right)\right] .
\end{aligned}
$$

The parameters of this distribution function are: the spatial beam variances $\sigma_{i}$, local momentum variances $\eta_{i}$ and momentum chirps $\gamma_{i}$, each in the transverse and longitudinal directions, that is $i=\{T, z\}$, respectively. Figure 1 shows these parameters in the context of the well-known (position and momentum) phase-space plot. Within the employed selfsimilar Gaussian pulse propagation approximation, the dynamics of these parameters are determined by the differential equations

$$
\begin{aligned}
& \frac{d \sigma_{i}}{d t}=\frac{2}{m_{e}} \gamma_{i}, \\
& \frac{d \gamma_{i}}{d t}=\frac{1}{m_{e}}\left(\eta_{i}+\frac{\gamma_{i}^{2}}{\sigma_{i}}\right)+\frac{1}{4 \pi \epsilon_{0}} \frac{N e^{2}}{6 \sqrt{\sigma_{i} \pi}} L_{i}(\xi), \\
& \frac{d \eta_{i}}{d t}=-\frac{2 \gamma_{i} \eta_{i}}{m_{e} \sigma_{i}},
\end{aligned}
$$

where $N$ is the number of electrons in the pulse, $\epsilon_{0}$ is the vacuum permittivity, and $e$ and $m_{e}$ are the charge and rest mass of the electron, respectively. In Eq. (2), the parameter

$$
\xi^{2} \equiv \frac{\sigma_{z}}{\sigma_{T}}, \quad \xi>0
$$

quantifies the ellipticity of the pulse, and the functions

$$
\begin{aligned}
& L_{T}(\xi) \equiv \frac{3}{2}\left(L(\xi)+\frac{\xi^{2} L(\xi)-\xi}{1-\xi^{2}}\right), \\
& L_{z}(\xi) \equiv \frac{3 \xi^{2}}{\xi-1}[\xi L(\xi)-1]
\end{aligned}
$$

depend on the function $L(\xi)$ defined by

$$
L(\xi) \equiv \frac{1}{2} \int_{0}^{\pi} \frac{d \theta}{1+\xi \sin (\theta)}
$$

which evaluates to a piecewise function that is well behaved for all physically meaningful (i.e., non-negative, real) $\xi$;

$$
L(\xi)=\left\{\begin{array}{cc}
\frac{\arcsin \left(\sqrt{1-\xi^{2}}\right)}{\sqrt{1-\xi^{2}}} & 0 \leq \xi \leq 1 \\
\frac{\ln \left(\xi+\sqrt{\xi^{2}-1}\right)}{\sqrt{\xi^{2}-1}} & \xi \geq 1
\end{array} .\right.
$$

This AG formalism of Michalik and Sipe ${ }^{1}$ employs a mean-field approximation in the evolution of the dynamical effect of the electron pulse's internal space-charge field. This is of course not strictly accurate for a Gaussian charge distribution and the error leads to a distortion of the pulse shape. Even so, the self-similar AG model of charge bunch dynamics has been benchmarked against particle tracking simulations for a number of electron pulse shapes, ${ }^{1,18}$ including the uniform ellipsoid which explicitly features a linear internal space-charge field. ${ }^{19}$ Indeed, as required by Liouville's Theorem, Eq. (2) can be reformulated to show that the product $\sqrt{\sigma_{i} \eta_{i}}$ is conserved in both the transverse $(i=T)$ and longitudinal $(i=z)$ directions; that is, the pulse emittance (proportional to the product of the position and momentum variance $^{21}$ ) is conserved in the AG formalism of electron pulse propagation. In the transverse dimension, this is consistent with the definition of "coherent fluence" employed by 
Reed et ll. $^{22}$ for time-resolved electron microscopy and, of course, the spatial emittance of an electron beam. ${ }^{20,21}$

\section{FREE-SPACE PROPAGATION DYNAMICS}

\section{A. The initial conditions}

For a DTEM or UED apparatus, where the electron pulse is produced in a laser-driven photo-electron gun, the implementation of the AG model of Michalik and $\mathrm{Sipe}^{1}$ requires knowledge of the initial $(t=0)$ bunch parameters immediately after generation at the photocathode surface inside the acceleration region of the gun. For the purposes of this paper, we will make a simple approximation to the behavior of the gun in order to provide generic initial conditions for the electron pulse.

For single-photon photoemission process with a spatially cylindrically symmetric incident Gaussian laser beam with Gaussian temporal irradiance profile

$$
I(x, y, t)=I_{0} \exp \left(-\frac{\left(x^{2}+y^{2}\right)}{w^{2}}-\frac{t^{2}}{\tau^{2}}\right),
$$

where $w$ is the half-width 1/e maximum (HW1/eM) of the spatial irradiance distribution, $\tau$ is the HW1/eM pulse duration, and $I_{0}$ is the peak pulse irradiance, the electron pulse's initial spatial variance is

$$
\sigma_{T}(0)=(\Delta x)^{2}=(\Delta y)^{2}=\frac{w^{2}}{2} .
$$

For the longitudinal direction, and in the limit where dispersion effects in the acceleration region of the electron gun can be neglected, we may write

$$
\sigma_{z}(0)=(\Delta z)^{2}=\frac{\left(v_{0} \tau\right)^{2}}{2}
$$

with the velocity of the pulse in the laboratory frame $v_{0}$ $=\sqrt{2 e V / m_{e} d}$, where $V$ and $d$ are the potential and cathodeanode separation of the gun's dc acceleration region, respectively. For spatially-uniform photoemission from a planar photocathode, we have $\gamma_{T}(0)=0$; i.e., there is no initial spatial momentum chirp in the electron bunch. Immediately after photo-generation, however, it is strictly inaccurate to say that $\gamma_{z}(0)=0$. This is because the excess photoemission energy $\Delta E=\hbar \omega-\Phi$, associated with the electron emission from a material with work function $\Phi$ using photons of energy $\hbar \omega$ (which gives the maximum initial velocity of an electron, $\left.v_{\max }=\sqrt{2 \Delta E / m_{e}}\right)$, can produce an initial momentum chirp. Nonetheless, the initial nonzero nature of $\gamma_{z}$ is quickly dominated by the evolution of the pulse, so this approximation will suffice in the region far from the gun.

The single-photon photoemission analysis of Jensen et $a l .{ }^{21}$ indicates that for a metallic photocathode the transverse momentum variance at $t=0$ is given by

$$
\left(\Delta p_{x}\right)^{2}=\left(\Delta p_{y}\right)^{2}=\eta_{T}(0) \approx \frac{m_{e} \epsilon_{F}(\hbar \omega-\Phi)}{\hbar \omega+\epsilon_{F}},
$$

in the limit where the Fermi energy $\epsilon_{F}$ of the metal photocathode and excess photoemission energy, $\Delta E=\hbar \omega-\Phi$ are significantly greater that the thermal energy $k_{B} T$ of the elec- trons in the photocathode-a condition that is generally met in laser-driven dc photoelectron guns. From a simplistic viewpoint, only the electrons generated with positive longitudinal momentum will contribute to the pulse since those electrons generated with negative momentum do not leave the photocathode. This implies that the uncertainty in the longitudinal direction is half that of the transverse directions; that is to say

$$
\left(\Delta p_{z}\right)^{2}=\left(\frac{\Delta p_{x, y}}{2}\right)^{2}
$$

so that

$$
\eta_{z}(0)=\frac{\eta_{T}(0)}{4}
$$

These initial conditions at $t=0$ for an incident Gaussian laser pulse of the form given in Eq. (7), will be used for the remainder of this paper.

$$
\begin{aligned}
& \sigma_{T}(0)=\frac{w^{2}}{2}, \\
& \sigma_{z}(0)=\frac{\left(v_{0} \tau\right)^{2}}{2}, \\
& \gamma_{T}(0)=0, \\
& \gamma_{z}(0)=0, \\
& \eta_{T}(0)=\frac{m_{e} \epsilon_{F}(\hbar \omega-\Phi)}{3\left(\hbar \omega+\epsilon_{F}\right)}, \\
& \eta_{z}(0)=\frac{\eta_{T}(0)}{4} .
\end{aligned}
$$

We recognize that these initial conditions for an electron pulse generated by a laser-driven dc gun are idealized in that they neglect several important effects; in particular, the interplay of dispersive $\left[\eta_{i}(0) \neq 0\right]$ and space-charge effects in the acceleration region of the photoelectron gun and transverse and longitudinal effects due to the divergence of the dc field in the vicinity of the gun anode. ${ }^{23,24}$ Such effects and the limits of the validity of Eq. (9), within the context of the AG model, will be presented elsewhere as they require a priori this presented extension of the AG model. However, Eq. (13) also represent a defined set of initial conditions that allows the effect of electron optics (i.e., magnetic lenses and rf cavities) on electron pulse propagation to be more readily understood. Moreover, they reflect the fact that the majority of dc photoelectron guns are driven by laser pulses with spatial and temporal profiles that are close to Gaussian., ${ }^{4,6}$ Only for specialized cases, such as the generation of uniform ellipsoid electron bunch shapes, is spatial and temporal manipulation of the incident laser pulse considered. ${ }^{19,25}$

\section{B. Initial electron bunch shape dependence}

The initial "space-time" eccentricity [Eq. (3)], $\xi(0)$ $=v_{0} \tau / \mathrm{w}$, of the photogenerated electron pulse plays a major 

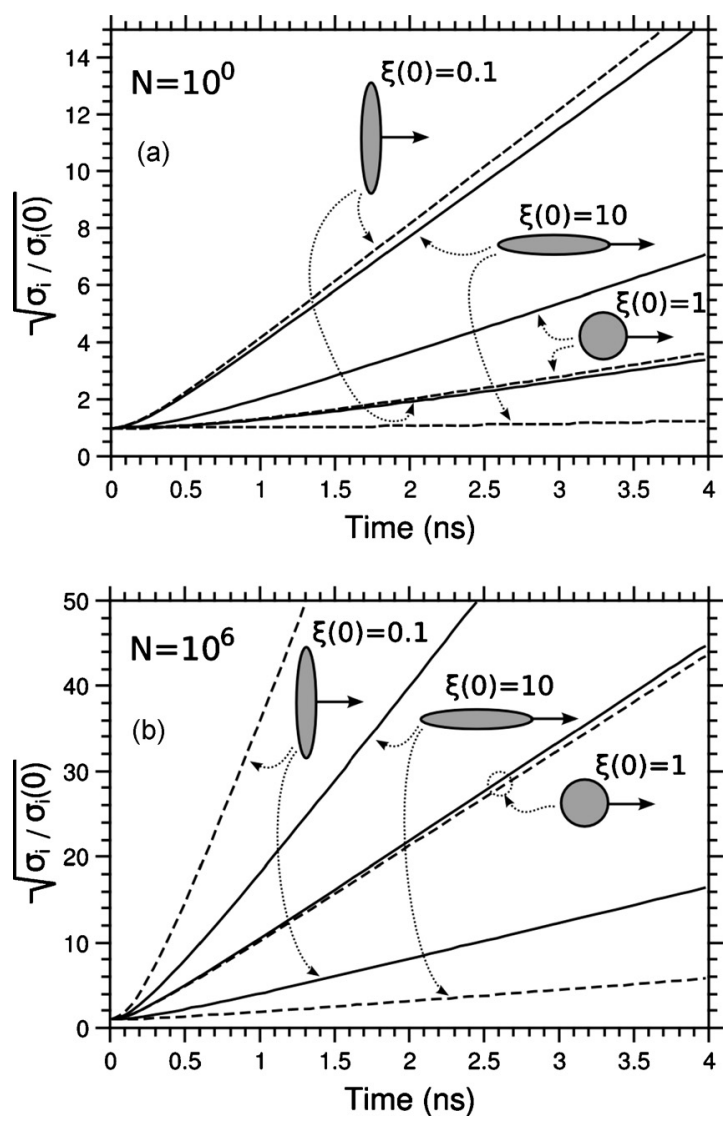

FIG. 2. Comparison of the propagation dynamics of disklike $[\xi(0)=0.1]$, spherical $[\xi(0)=1]$ and cigarlike $[\xi(0)=10]$ pulses, generated from a $261 \mathrm{~nm}$ laser driven Ta photocathode in a $20 \mathrm{kV}$ dc electron gun, at (a) negligible charge densities $\left(N=10^{\circ}\right)$ and (b) charge densities suitable for single shot UED studies $\left(N=10^{6}\right)$ : the normalized transverse beam width $\left[\sqrt{\sigma_{T} / \sigma_{T}(0)}\right]$ (solid line) and normalized longitudinal pulse width $\left[\sqrt{\sigma_{z} / \sigma_{z}(0)}\right]$ (dashed line).

role in the bunch propagation dynamics, particularly when space-charge effects dominate. Figure 2 illustrates the typical dynamics of initially spherical $\left[\sigma_{T}(0)=\sigma_{z}(0)\right]$, "disklike" $\left[\sigma_{T}(0) \gg \sigma_{z}(0)\right]$, and "cigarlike" $\left[\sigma_{z}(0) \gg \sigma_{T}(0)\right]$ pulses. In all cases, the same initial conditions of Eq. (13) are employed, assuming a Ta photocathode $\left(\epsilon_{F}=5.3 \mathrm{eV}, \Phi=4.25 \mathrm{eV}\right)$ illuminated by $261 \mathrm{~nm}$ laser radiation $(\hbar \omega=4.75 \mathrm{eV}) .^{23}$ The initial bunch dimensions are given by the ellipticities $\xi(0)$ $=\{0.1,1,10\}$ and the HW1/eM bunch widths $\sqrt{2 \sigma_{T}(0)}$ $=\sqrt{2 \sigma_{z}(0)}=100 \mu \mathrm{m}$ for the spherical case, corresponding to a $\sim 1$ ps duration electron pulse (for $v_{0} \approx c / 3$ from a dc photogun with $V=30 \mathrm{kV}$ ). The initial bunch "volume," proportional to $\sigma_{T}(0) \sqrt{\sigma_{z}(0)}$, is the same in all cases; so that the initial peak pulse charge density is six orders of magnitude greater in Fig. 2(b) $\left(N=10^{6}\right.$ electrons in the bunch) than in Fig. 2(a) $\left(N=10^{\circ}\right)$. For $100 \mathrm{keV}(20 \mathrm{keV})$ electrons, for which $v_{0}=0.55 c\left(v_{0}=0.28 c\right)$, the 4 ns time of flight in Fig. 2 corresponds to a propagation distance of $66 \mathrm{~cm}(34 \mathrm{~cm})$.

The bunch propagation dynamics for negligible pulse charge density $\left(N=10^{\circ}\right)$ shown in Fig. 2(a) display the trends expected from the initial photoemission conditions. In particular, in the initially spherical case $\left[\sigma_{T}(0)=\sigma_{z}(0)\right]$, the transverse bunch width $\sqrt{2 \sigma_{T}}$ expands twice as rapidly as the longitudinal length $\sqrt{2 \sigma_{z}}$ due to the factor of two difference in the initial momentum variances $\Delta p_{i}$ and the zero initial pulse chirp $\left(\gamma_{i}=0\right)$. Naturally, for initially nonspherical electron bunches, the pulse propagation model also shows that the relative bunch broadening $\sqrt{\sigma_{i} / \sigma_{i}(0)}$ for comparable $\eta_{i}$ is greatest for the smallest initial $\sigma_{i}(0)$; that is both disklike and cigarlike pulses are initially driven toward the spherical regime. In all cases, this intrinsic electron pulse broadening due to nonzero $\eta_{i}$ generates a momentum chirp across the electron bunch; in the longitudinal dimension the faster electrons photoemitted with larger excess energy leading the slower electrons.

For an electron pulse with a higher initial space-charge density, intrapulse Coulomb effects can dominate the propagation dynamics. In Fig. 2(b), we display the predicted temporal dynamics of electron pulses with the same initial size and photoemission conditions as Fig. 2(a) but with $N=10^{6}$ electrons - the number required for single-shot diffraction pattern measurements in a DTEM. ${ }^{12,23}$ The significant influence of space-charge effects for the initial peak pulse charge density of $0.0287 \mathrm{C} / \mathrm{m}^{3}$ is clearly evident. In the spherical case, space-charge induced bunch broadening overwhelms the initial difference between $\eta_{T}$ and $\eta_{z}$, producing an almost uniform three-dimensional 'Coulomb explosion' of the electron pulse with a one-dimensional bunch broadening rate roughly one order of magnitude greater than that for $N$ $=10^{0}[$ Fig. $2(\mathrm{a})]$. For $\xi(0)>1[$ or $\xi(0)<1]$, the smaller initial transverse (longitudinal) bunch dimension experiences the greatest relative expansion in free-space propagation. This is to be expected, because the initial $\eta_{i}$ are nonzero (but comparable in magnitude) while Coulomb effects will produce differential pulse broadening in the dimension with the largest internal space-charge field (i.e., the dimension with the largest electrostatic potential gradient). In fact, both the $N$ $=10^{6}$ disklike and cigarlike pulse examples shown in Fig. 2(b) transition through a spherical bunch shape $(\xi \approx 1)$ after a time of flight of $\sim 0.5 \mathrm{~ns}$, at which point the peak pulse charge density has already been reduced by a factor of $\sim 100$. The initial impulse induced by the strong space-charge field then provides for continued linear expansion (in time), resulting in a linear pulse chirp in the dimension associated with the smallest initial HW1/eM bunch width ${ }^{1,23} \sqrt{2 \sigma_{i}(0)}$.

The data presented in Fig. 2 using the AG model of Michalik and Sipe $^{1}$ are entirely consistent with prior works. $^{20,26}$ In femtosecond UED experiments, significant efforts are made to offset space-charge induced temporal (longitudinal, z) pulse broadening by reducing the time of flight from the gun photocathode to the sample..$^{20,22}$ Moreover, efforts are now underway to compensate for temporal electron pulse broadening using rf cavity pulse compression techniques. ${ }^{16}$ In UEM, employing a standard retrofitted electron microscope column driven by megahertz repetition-rate subpicosecond lasers, ${ }^{15}$ deleterious space-charge effects have obliged operation in the $\sim 1$ electron/pulse regime to maintain high spatial resolution. Even for nanosecond DTEM [the extreme cigarlike pulse $(\xi \gg 1)]$, transverse space-charge induced beam broadening has necessitated the insertion of an additional magnetic electron lens in a microscope column to allow more efficient delivery of electrons to the specimen when $N>10^{6} .^{27}$ In this paper, however, we will restrict the 
discussion to ultrashort (picosecond and subpicosecond) electron pulses which (i) allow for the temporal resolution of fundamental events occurring on ultrafast time scales in materials science, biology and chemistry ${ }^{2}$ and (ii) can be generated in the disklike regime $(\xi<1)$ where transverse bunch broadening effects are minimized (Fig. 2) (enabling more efficient beam propagation) and rf cavities can be used to compensate and reverse longitudinal (temporal) pulse broadening. ${ }^{17}$

\section{EXTENSION TO INCLUDE EXTERNAL FORCES}

Simulation of electron pulse propagation in UEM and modern UED apparatus ${ }^{16,20,26}$ requires the ability to model electron-optical elements designed to control the threedimensional space-charge and dispersion induced broadening of the pulse, and hence deliver electron pulses of the desired quality to the specimen. In this section, we show that the benchmarked $^{1,18}$ analytical electron pulse propagation analysis of Michalik and Sipe ${ }^{1}$ can be extended to include external forces on the pulse, such as the influence of magnetic electron lenses and rf pulse compression cavities. ${ }^{16,17}$

It is a straightforward exercise to show, due to superposition, that adding an additional general force term to the Coulomb force in the previous derivation, does not affect the Coulomb interaction and allows one to use the framework already established. In Ref. 1, the authors reconstruct the coefficients of the coordinate products of the distribution function defined in Eq. (1) as a matrix A, so that Eq. (1) becomes

$$
f=\frac{N}{(2 \pi)^{3} \sigma_{T} \eta_{T} \sqrt{\sigma_{z} \eta_{z}}} \exp ^{-(1 / 2) U^{T} A U} .
$$

where

$$
\begin{aligned}
& \{j, k\}=\{1,2,3,4,5,6\}, \\
& U_{j} \equiv\left\{x, p_{x}, y, p_{y}, z, p_{z}\right\} .
\end{aligned}
$$

All of the differential equations in Eq. (1) relate to the time derivative of the inverse of the matrix $A$. If we choose to call the resultant matrix $K$, we can write the decomposition as,

$$
\frac{\partial}{\partial t}\left(A^{-1}\right)_{j k} \equiv K_{j k}=K_{j k}^{f l o w}+K_{j k}^{f o r c e}+K_{j k}^{e x t},
$$

where the "flow" (geometric motion or equivalently "Gaussian beam optics" for bosons) and "force" (internal Coulomb repulsions) terms are described in Ref. 1, and we now define the 'external' term as

$$
K_{j k}^{e x t} \equiv \frac{1}{N} \int U_{j} U_{k} \vec{F}_{e x t} \cdot\left(\vec{\nabla}_{p} f\right) d^{3} x d^{3} p,
$$

with

$$
\vec{\nabla}_{p} \equiv \frac{\partial}{\partial p_{x}} \hat{x}+\frac{\partial}{\partial p_{y}} \hat{y}+\frac{\partial}{\partial p_{z}} \hat{z} .
$$

We expect each of these $K$ matrices to be symmetric with respect to the interchange of indices. The resultant differential equations for the $T$ (or $z$ ) directions are then given by elements of $K_{j k}$ as

$$
\begin{aligned}
& \frac{d \sigma_{T(z)}}{d t}=K_{11(55)}=\left(\frac{d \sigma_{T(z)}}{d t}\right)_{F_{e x t}=0}+K_{11(55)}^{e x t}, \\
& \frac{d \gamma_{T(z)}}{d t}=K_{12(56)}=\left(\frac{d \gamma_{T(z)}}{d t}\right)_{F_{e x t}=0}+K_{12(56)}^{e x t}, \\
& \frac{d \eta_{T(z)}}{d t}=\left(\frac{d \eta_{T(z)}}{d t}\right)_{F_{e x t}=0}+\frac{\gamma_{T(z)}^{2}}{\sigma_{T(z)}^{2}} K_{11(55)}^{e x t},
\end{aligned}
$$

where the first terms on the right hand side of Eq. (17) are Eqs. (2a)-(2c), respectively; that is, they reflect the pulse dynamics in the absence of an external force $\left(F_{\text {ext }}=0\right)$.

One might have expected the $K_{22(66)}$ terms to appear in the differential equation for the time derivative of $\eta_{T(z)}$ in Eq. (17c). In fact, it can be shown that no resultant $K_{22(66)}$ will directly affect these equations. This information does, however, provide a mathematical check on the physical validity of the new matrix: For any force term $f$ (i.e., the internal coulomb force force 1 or any external force "ext"), it is known that the $K^{f}$ matrix components must satisfy the relation

$$
K_{12(56)}^{f}=K_{21(65)}^{f}=\frac{\sigma_{T(z)}}{2 \gamma_{T(z)}} K_{22(66)}^{f} .
$$

For constant static forces having no dependence in $x, y$, $z$, or $t$, one can readily show that there is no effect on the pulse propagation parameters $\sigma_{i}, \eta_{i}$, and $\gamma_{i}$. For example, consider a field $\vec{E}=-E \hat{z}$ such that electrons are accelerated in the positive $z$ direction by $\vec{F}_{\text {ext }}=q E \hat{z}=q(V / d) \hat{z}$, so that from Eq. (16) we have

$$
K_{j k}^{e x t}=\frac{1}{N} \frac{q V}{d} \int U_{j} U_{k}\left(\frac{p_{z}}{\eta_{z}}-\frac{\gamma_{z} z}{\sigma_{z} \eta_{z}}\right) f d^{3} x d^{3} p ;
$$

but it is clear that no function of this form multiplying $f$, which is a Gaussian distribution, can avoid creating an odd integrand, and thus $K_{j k}^{e x t}=0$ for all $j$ and $k$ (or equivalently $\left.K^{e x t}=\hat{0}\right)$ meaning that this static field has no effect on the parameters of the pulse. Of course, this is only true in the pulse frame, where there is no total momentum and thus $\Delta E=(\Delta p)^{2} / 2 m_{e}$ is unchanged. However, in the laboratory frame, where $\Delta E=p \Delta p / m_{e}$, there is an increase in the momentum spread, as the pulse now has the large momentum $p$ imparted by the accelerating field.

\section{MAGNETIC LENSES AND RF CAVITIES}

External forces that vary with position in the pulse reference frame (i.e., are not constant) act to change the electron pulse propagation dynamics, most often by affecting $\gamma_{i}$ - the spatial dependence of the local relative mean electron momenta. Below, we consider two examples of electron optical elements that are being used, or are under consideration, for controlling and manipulating the space-time dynamics of electron pulses in DTEM and UED instrumentation; namely, magnetic lenses for spatial focusing and $\mathrm{rf}$ pulse compression cavities. 


\section{A. The parabolic "perfect" lens}

For the case of a perfect lens, or in the longitudinal direction a perfect $\mathrm{rf}$ compression element, with a parabolic potential, the external force is linear with distance from the center of the electron pulse:

$$
\vec{F}_{e x t}=-M_{T} r \hat{\mathrm{r}}-M_{z} z \hat{\mathrm{z}} .
$$

Here, $M_{T}$ and $M_{z}$ characterize the strength of a lens or a compressor, respectively, and are chosen to be positive for pulse focusing. Substitution into the integral equation for the $K^{\text {ext }}$ matrix elements,

$$
\begin{aligned}
K_{j k}^{e x t} \equiv & \frac{1}{N} \int U_{j} U_{k}\left(\vec{\nabla}_{p} f\right) \cdot \vec{F}_{e x t} d^{3} x d^{3} p \\
= & \frac{1}{N} \int U_{j} U_{k}\left[x\left(\frac{p_{x}}{\eta_{T}}-\frac{\gamma_{T} x}{\sigma_{T} \eta_{T}}\right)+y\left(\frac{p_{y}}{\eta_{T}}-\frac{\gamma_{T} y}{\sigma_{T} \eta_{T}}\right)\right. \\
& \left.+z\left(\frac{p_{z}}{\eta_{z}}-\frac{\gamma_{z} z}{\sigma_{z} \eta_{z}}\right)\right] f d^{3} x d^{3} p,
\end{aligned}
$$

leads to the $6 \times 6$ matrix

$$
K^{e x t}=\left(\begin{array}{ccc}
\hat{M}_{T} & \hat{0} & \hat{0} \\
\hat{0} & \hat{M}_{T} & \hat{0} \\
\hat{0} & \hat{0} & \hat{M}_{z}
\end{array}\right),
$$

where

$$
\hat{M}_{i}=\left(\begin{array}{cc}
0 & M_{i} \sigma_{i} \\
M_{i} \sigma_{i} & 2 M_{i} \gamma_{i}
\end{array}\right) .
$$

This matrix adds terms to the unforced differential equations by use of Eq. (17), so that the set analogous to Eq. (2) become

$$
\begin{aligned}
& \frac{d \sigma_{i}}{d t}=\frac{2}{m_{e}} \gamma_{i}, \\
& \frac{d \gamma_{i}}{d t}=\frac{1}{m_{e}}\left(\eta_{i}+\frac{\gamma_{i}^{2}}{\sigma_{i}}\right)+\frac{1}{4 \pi \epsilon_{0}} \frac{N e^{2}}{6 \sqrt{\sigma_{i} \pi}} L_{i}(\xi)+M_{i} \sigma_{i}, \\
& \frac{d \eta_{i}}{d t}=-\frac{2 \gamma_{i} \eta_{i}}{m_{e} \sigma_{i}} .
\end{aligned}
$$

We note that the quantity $\sqrt{\sigma_{i} \eta_{i}}$ (the pulse emittance) is still conserved in the description of electron pulse propagation in the AG model even after the addition of the extra force term(s) in Eq. (24b) due to the external linear force. This is, of course, true in general since a linear force (like the mean-field approximation for space-charge effects) will only directly affect the pulse's momentum chirp $\gamma_{i}$ and not initially either its size $\sigma_{i}$ or local momentum variance $\eta_{i}$, although the latter are coupled to the change in $\gamma_{i}$ [as shown in the AG model in Eqs. (24a) and (24c)].

To specify the physical region of influence of the lenses, it is convenient to redefine $M_{i}$ to include a super-Gaussian envelope in $z^{\prime}$, the position of the peak of the pulse in the laboratory frame;
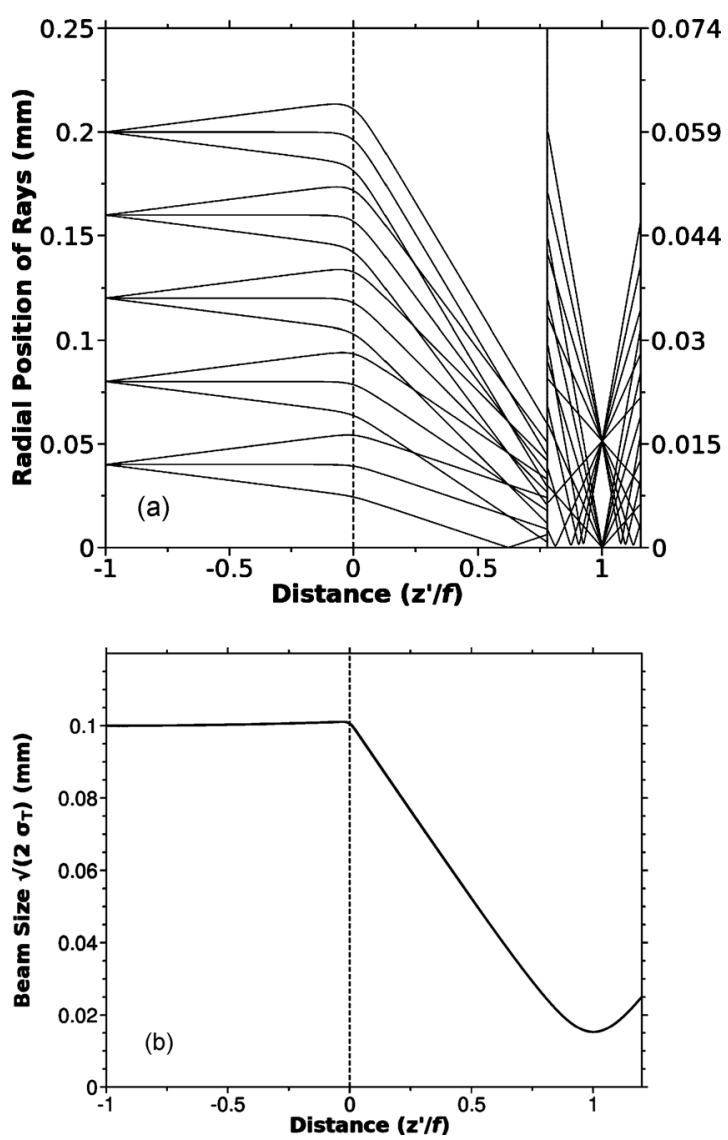

FIG. 3. Comparison of the focal behavior of an $f=63.5 \mathrm{~mm}$ aberration-free lens in (a) the kinematic electron trajectory model and (b) the AG model.

$$
M_{i} \rightarrow M_{i} \exp \left[-\left(\frac{z^{\prime}-z_{\text {lens }}^{\prime}}{L_{\text {lens }} / 2}\right)^{2 n}\right]
$$

where $z_{\text {lens }}^{\prime}$ is the position of the center of the lens, $L_{\text {lens }}$ is the length of the lens, and $n$ is the super-Gaussian order parameter. The order parameter defines the "sharpness" of the edge of the region of influence with integer values ranging from 1 (a simple Gaussian) through $\infty$ (a top-hat). We connect $z^{\prime}$ to $t$ through $v_{0}$; the kinetic equations are simply defined for the position of the peak of the pulse.

Figure 3 compares the spatial pulse focusing induced by the magnetic field of a single current loop (diameter of 12.7 $\mathrm{mm}(0.5$ in. $)$ at $\left.z^{\prime}=0\right)$ calculated kinematically, with that predicted by Eq. (24) in the absence of space-charge effects $(N=0)$. Figure 3(a) displays the radial position from the lens axis of selected $20 \mathrm{kV}$ electrons (velocity $v_{0}=8.4$ $\times 10^{7} \mathrm{~m} / \mathrm{s}$ ) evaluated from a full kinematic description of the effect of the loop's magnetic field ${ }^{28}$ on the individual electron trajectories for a lens of focal length $f=63.5 \mathrm{~mm}$ (corresponding to a total loop current of 450 A). For clarity, only selected electron trajectories with an initial beam divergence of $\theta= \pm 0.24 \mathrm{mrad}$ (and $0 \mathrm{mrad}$ ) at $z^{\prime}=-f=$ $-63.5 \mathrm{~mm}$ are shown, resulting in the expected radial focal spot size of $f \cdot \theta=15 \mu \mathrm{m}$. For this beam divergence, one may determine an initial transverse momentum variance $\eta_{T}(0)$ $=\left(m_{e} v_{0} \theta\right)^{2} / 2$ for comparison with the pulse propagation model. Figure 3(b) presents the results obtained for $\sqrt{2 \sigma_{T}}$ using Eq. (24) and this initial value of $\eta_{T}, \gamma_{T}=0$, an initial 

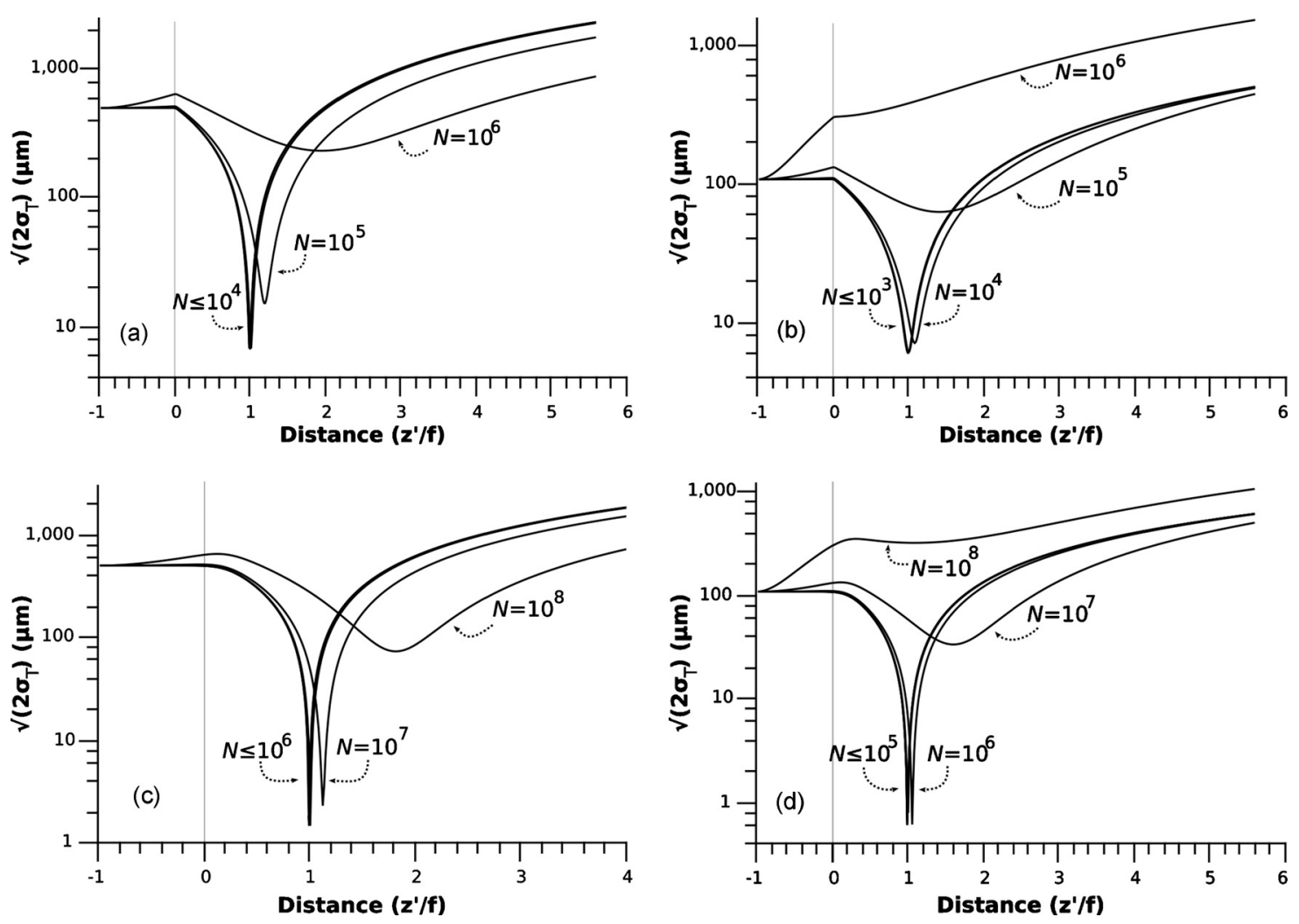

FIG. 4. Focal behavior of perfect magnetic lenses predicted by the AG model (with dc acceleration voltage of $20 \mathrm{kV}$ ) as a function of pulse charge $N$ : (a) $f=63.5 \mathrm{~mm}$ and disklike $[w=500 \mu \mathrm{m}$ and $\xi(0)=0.1]$; (b) $f=63.5 \mathrm{~mm}$ and cigarlike $[w=107.7 \mu \mathrm{m}$ and $\xi(0)=10]$; (c) $f=6.35 \mathrm{~mm}$ and disklike $[w$ $=500 \mu \mathrm{m}$ and $\xi(0)=0.1]$; (d) $f=6.35 \mathrm{~mm}$ and cigarlike $[w=107.7 \mu \mathrm{m}$ and $\xi(0)=10]$. A logarithmic scale is used for comparative clarity.

Gaussian electron beam waist size $w=\sqrt{2 \sigma_{T}}=0.1 \mathrm{~mm}$ [i.e., a beam size consistent with the trajectories shown in Fig. 3(a)], and the value of $M_{T}$ adjusted to produce a focal spot at $z^{\prime}$

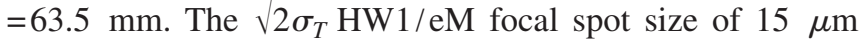
predicted by the AG pulse propagation model is clearly in very good agreement with that determined from the full electron trajectory calculation. In obtaining the results presented in Fig. 3(b), we have used $n=1$ and $L_{\text {lens }}=2.54 \mathrm{~mm}(0.1 \mathrm{in}$.) to provide for a good approximation for the lensing action of the Lorentzian magnetic field distribution of the current loop ${ }^{29}$ in the AG model.

We note that the use of a small beam diameter $(<0.5 \mathrm{~mm})$ compared to the current loop diameter in the trajectory calculations of Fig. 3(a) ensures that the spherical lens aberrations are negligible, as evidenced by the highquality focal image at $z^{\prime} / f=1$. For incident electron beam diameters $\sqrt{2 \sigma_{T}}$ greater than about $1 \mathrm{~mm}$ (one tenth of the current loop diameter), the kinematic particle trajectory analysis indicates that the magnetic lens has significant spherical aberration - an expected result for a single current loop with no pole pieces. ${ }^{28}$ For example, a beam with initial radial axial beam displacements of up to $2 \mathrm{~mm}$ incident on the $f=63.5 \mathrm{~mm}$ focal length magnetic current loop lens of Fig. 3 produces an effective focal point (circle of least confusion) that is shifted to $z^{\prime}=0.98 f$ (i.e., $1.3 \mathrm{~mm}$ toward the magnetic lens) due to positive spherical aberration. In principle, the AG model can accommodate such a spherical aberration through the inclusion of an external nonlinear force term proportional to $r^{3} \hat{r}$ in Eq. (20), yielding an additional term proportional to $\sigma_{T}^{2}$ in Eq. (24b). However, this disre- gards the mean-field self-similar Gaussian approximation inherent in the AG model as a nonlinear external force would cause pulse breakup.

\section{B. Focusing: Space-charge effects}

In the transverse spatial dimension, space-charge effects that increase $\sigma_{T}$ can be compensated for by using magnetic electron lenses. ${ }^{16,27}$ In an UEM, of course, the electron pulse will also need to be focused onto the specimen-the focal spot size, post specimen image magnification, and chargecoupled device detector pixelation determining the spatial resolution in a TEM geometry. ${ }^{23}$ The extent to which spacecharge effects limit the focal fidelity is expected to be dependent upon both $N$ and the pulse shape (i.e., the pulse's spacetime eccentricity).

Figure 4 shows the predicted dynamics of the spatial electron pulse waist (HW1/eM) for both disklike and cigarlike pulses under perfect focusing for lenses of two focal lengths; a "long" focal length of $63.5 \mathrm{~mm}$ [Figs. 4(a) and 4(b)] and a "short" focal length of $6.35 \mathrm{~mm}$ [Figs. 4(c) and 4(d)]. In all cases, the pulse is assumed to be accelerated to $20 \mathrm{kV}$ and generated from a Ta photocathode with a negligible excess photoemission energy (i.e., $\eta_{i} \rightarrow 0$ ) in order to highlight the effect of space-charge. For both lens strengths, the deleterious effects of space-charge are evident as the number of electrons/pulse $N$ is increased. The single-electron $(N=1)$ focal spot sizes of $\sim 6$ microns for the $f=63.5 \mathrm{~mm}$ lens and $\sim 0.6$ microns for the shorter $f=6.35 \mathrm{~mm}$ lens increase markedly for $N \gtrsim 10^{5}$ and $N \gtrsim 10^{6}$, respectively. This 
order of magnitude difference is fundamentally related to the greater time of flight before the focus of the $f=63.5 \mathrm{~mm}$ lens; it increases the impulse that the internal Coulomb force can exert on the pulse as its charge density is increased under focusing. A consequent further effect is a shift in the focal position to $z>f$. A more subtle effect is that disklike pulses are more readily focused than cigarlike pulses - the rate of transverse pulse broadening due to space-charge effects already being intrinsically greater in the latter (Fig. 2). Thus, the electron pulse propagation model predicts that higher fidelity focusing will be achieved with shorter focal length magnetic lenses, higher acceleration energy electrons (time of flight reduction), and pulses with $\sigma_{T} \gg \sigma_{z}$ at the entrance aperture of the lens.

\section{C. rf cavities}

In the longitudinal $(z)$ direction, a resonant $\mathrm{rf}$ cavity can be used in a UEM column or UED experiment ${ }^{16,30}$ to act as a pulse compression or "temporal focusing" element. We could choose to employ any of the many possible resonant cavity structures, ${ }^{16,31}$ but for simplicity we will consider only the evacuated cylindrical (or "pillbox") $\mathrm{TM}_{010}$ cavity for which the electrons propagate down the axis. ${ }^{30,31}$ The known mathematical forms of the electric and magnetic components of this resonant mode together with a super-Gaussian envelope function [Eq. (25)] allow the three main effects of a pillbox rf cavity element on the electron pulse to be described within the approximation of the linear force extension to the AG model: the primary action of the pulse compression (or acceleration and expansion dependent upon the relative rf phase), and the transverse lens effects due to the magnetic component of the $\mathrm{TM}_{010}$ resonance and the electric rf cavity fringe fields at its entrance and exit apertures. ${ }^{32}$

The spatial dependence of the axial rf electric field experienced by the electron pulse in a $\mathrm{TM}_{010}$ cavity of radius $a$ may be written as

$$
E\left(r, z, z^{\prime}\right)=E_{0} \sin \left(\frac{\Omega\left(z^{\prime}-z\right)}{v_{0}}+\phi\right) \mathrm{J}_{0}\left(\frac{k r}{a}\right),
$$

where $E_{0}$ is the oscillating field amplitude, $\Omega$ is the rf frequency, and $\phi$ is a phase factor determining whether the rf cavity acts as a pulse compressor $(\phi \approx 0)$ or an electron accelerator $(\phi \approx \pm \pi / 2)$. The coordinate $z^{\prime}$ describes the position of the center of the pulse in the rf cavity, which extends from $z^{\prime}=-d / 2$ to $z^{\prime}=d / 2$, and $z$ is the position of any electron in the pulse with respect to the center of the pulse. For the $\mathrm{TM}_{010}$ oscillation mode, the rf frequency is given by

$$
\Omega=\frac{c k}{a}
$$

where $c$ is the speed of light in a vacuum and $k=2.405$ is the first zero of the $\mathrm{J}_{0}$ Bessel function.

For the usual case of a spatially compact electron pulse with respect to the dimension of the rf cavity (i.e., $\Omega z \ll v_{0}$ and $\sqrt{\sigma_{T}} \ll a$ ), Eq. (26) can be rewritten as

$$
E\left(r, z, z^{\prime}\right)=E_{0}\left[\sin \left(\frac{\Omega z^{\prime}}{v_{0}}+\phi\right)-\frac{\Omega z}{v_{0}} \cos \left(\frac{\Omega z^{\prime}}{v_{0}}+\phi\right)\right]
$$

which demonstrates that the rf cavity acts both as a pulse acceleration [first term on RHS of Eq. (28)] and a pulse compression or expansion [second term on RHS of Eq. (28)] device. The second term then gives the desired form [see Eq. (20)] for the force manipulating the temporal dynamics and characteristics during electron pulse compression;

$$
\vec{F}_{z, \mathrm{rf}}=-\left[\frac{e \Omega E_{0}}{v_{0}} \cos \left(\frac{\Omega z^{\prime}}{v_{0}}+\phi\right)\right] z \hat{\mathrm{z}} \text {. }
$$

By considering the impulse exerted by the rf cavity field on the electrons in the pulse, it is straightforward to show that pulse compression (when $\phi=0$ ) is maximized for $\Omega d /\left(2 v_{0}\right)=\pi / 2$; that is, the time of flight of the electron pulse through the rf cavity of length $d$ is equal to half the rf period. Such a calculation assumes that the pulse velocity $v_{0}$ is constant; so that, the condition that $\phi=0$ when the pulse is at the center of the rf cavity (i.e., when the pulse is at $z^{\prime}$ $=0)$ is readily met. As suggested by the acceleration term in Eq. (28), this is only a good approximation if the rf field amplitude $E_{0}$ is sufficiently small to not affect the velocity of the peak of the pulse as it propagates through the cavity. As a result, in practice, the phase $\phi$ of the rf field is adjusted to optimize the performance of the cavity.

The two main effects contributing to the transverse lens of a $\mathrm{TM}_{010}$-mode rf cavity, its magnetic field component and electric fringe field aperture effects, may also be included within the linear force approximation. The azimuthal magnetic component of the resonant mode oscillates $90^{\circ}$ out of phase with the electric component and with a $\mathrm{J}_{1}$ Bessel radial form; i.e., $\mathrm{J}_{1}(k r / a) \sim k r / a$ for small $r$. Symmetry and Gauss' law dictate that in the vicinity of the axial center of the entrance and exit apertures of the rf cavity there exists a radial component of the oscillating electric field determined by $E_{r}=-(r / 2)\left(\partial E_{z} / \partial z\right)_{r=0},{ }^{32}$ where the form of the axial electric field for $\mathrm{TM}_{010}$ oscillation [Eq. (26)] implies that, in the context of this analysis, its axial gradient is solely dependent upon the envelope function for the rf cavity [Eq. (25)]. In practice, of course, the exact form of $E_{r}(z)$ is strongly tied to the size and shape of the rf cavity apertures, as illustrated by 1.5-cell rf photogun designs. ${ }^{33}$ For the case of an evacuated cavity, these two contributions result in a net force,

$$
\begin{aligned}
\vec{F}_{r, \mathrm{rf}}= & e E_{0}\left[\frac{v_{0} \Omega}{c^{2}} \cos \left(\frac{\Omega z^{\prime}}{v_{0}}+\phi\right)\right. \\
& \left.+\frac{2 n}{d}\left(\frac{z^{\prime}-z_{\mathrm{rf}}^{\prime}}{d / 2}\right)^{2 n-1} \sin \left(\frac{\Omega z^{\prime}}{v_{0}}+\phi\right)\right] r \hat{\mathrm{r}} .
\end{aligned}
$$

In obtaining Eq. (30), which is also of the form for use in Eq. (20), we have again used an arbitrary super-Gaussian of order $n$ as a region of influence envelope [see Eq. (25)] with $L_{\mathrm{rf}} \equiv d$, the rf cavity length. For the examples shown below under the pulse compression conditions $(\phi=0$ when the pulse is at $z^{\prime}=0$ ), both effects are defocusing for the electron pulse and the magnetic contribution is smaller than the transverse lenses associated with the rf cavity apertures when $d$ 

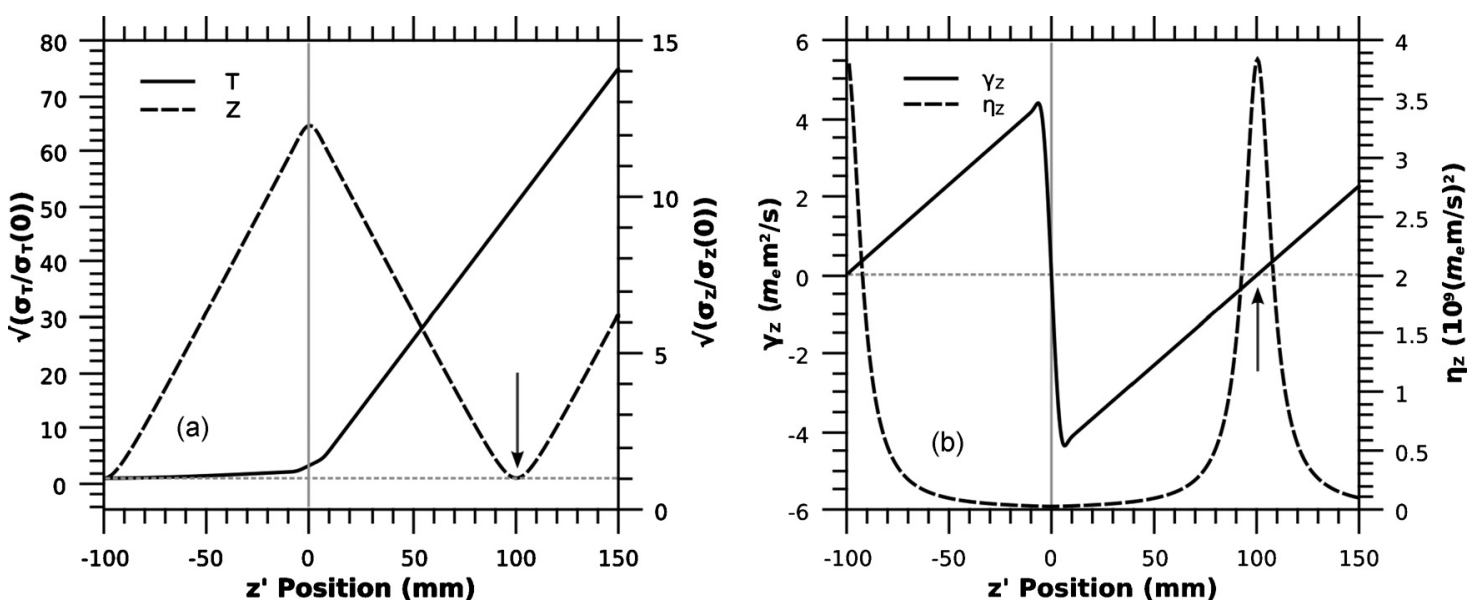

FIG. 5. Temporal focusing of an rf pulse compression cavity simulated by the AG model in the absence of space-charge effects: dependences of (a) the normalized pulse widths $\left[\sqrt{\sigma_{i} / \sigma_{i}(0)} ; T=\right.$ solid line, $z=$ dashed line $]$ and (b) $\gamma_{z}$ (solid line) and $\eta_{z}$ (dashed line, right axis) as a function of position from the 20 $\mathrm{kV}$ Ta dc photogun $\left(z^{\prime}=-100 \mathrm{~mm}\right)$, through the rf cavity $\left(z_{\mathrm{rf}}^{\prime}=0\right)$, and beyond the temporal focus (at $z^{\prime}=100 \mathrm{~mm}$ ). Initial laser pulse parameters: $w$ $=100 \mu \mathrm{m}$ and $\tau=100$ fs [i.e., $\xi(0) \approx 0.084]$. rf cavity parameters: $E_{0}=423.5 \mathrm{kV} / \mathrm{m}$ and $\phi=0$. The arrow indicates the position of the temporal focus.

$=\pi v_{0} / \Omega,{ }^{32}$ even though its magnitude is maximized for $\phi$ $=0$. Other effects, such as magnetic fringe field aperture effects, could also be included but are significantly weaker.

Figures 5 and 6 illustrate the operation of a $\Omega / 2 \pi$ $=3 \mathrm{GHz} \mathrm{TM}_{010}$ rf cavity of axial length $d=\pi v_{0} / \Omega$ as an electron pulse compressor tuned to produce the shortest pulse at a distance $z^{\prime}=10 \mathrm{~cm}$ behind the cavity. In both cases, the initial conditions for the photo-generated pulse at $z^{\prime}=-10 \mathrm{~cm}$ are the same; a $20 \mathrm{kV}$ Ta photocathode $(\Phi$ $=4.25 \mathrm{eV})$ irradiated by pulsed $4.75 \mathrm{eV}(261 \mathrm{~nm})$ laser radiation with $w=0.1 \mathrm{~mm}$ and $\tau=100 \mathrm{fs}$. The electron pulse is therefore initially in the disklike regime $\left[\sigma_{T}(0) \gg \sigma_{z}(0)\right]$, for which space-charge effects predominantly act in the longitudinal direction, broadening the pulse in time (see Fig. 2).

Figure 5 depicts the optimized rf cavity $\left(L_{\mathrm{rf}}=d\right.$ $=14 \mathrm{~mm}, z_{\mathrm{rf}}^{\prime}=0$, and $n=2$ [in Eq. (25)] to reflect the sharper end to the region-of-influence of the rf cavity) performance with $N=1$ electrons in the pulse; conditions under which the internal pulse space-charge effects are negligible, so that the rf cavity only compensates for the "dispersive" electron pulse broadening to $\sim 1.3 \mathrm{ps}$ at the rf cavity entrance [Fig. $5(\mathrm{a})]$ that is due to the initial longitudinal momentum spread caused by the $\Delta E=0.5 \mathrm{eV}$ excess photoemission energy. For an rf field amplitude $E_{0}=423.5 \mathrm{kV} / \mathrm{m}$ and phase $\phi=0$, the dispersion-generated momentum chirp $\gamma_{z}$ is exactly reversed [Fig. 5(b)] upon propagation through the rf cavity to produce a compressed pulse at $z^{\prime}=10 \mathrm{~cm}$ behind the rf cavity [Fig. 5(a)]. In this case, the pulse is returned to its original temporal duration-an exact 1-to-1 "temporal imaging" of the photogenerated electron pulse at $z^{\prime}=-10 \mathrm{~cm}$ by the rf cavity to $z^{\prime}=10 \mathrm{~cm}$. On the other hand, the dynamics of the spatial size of the electron pulse are clearly dominated by the negative compound transverse lens of the rf cavity, increasing at the temporal focus to a factor of $50 \times$ its initial value of $\sqrt{\sigma_{T}}=71 \mu \mathrm{m}$.

In analogy to optical imaging, the compressed pulse duration in this dispersive limit is determined by the image-toobject distance (magnification) ratio, with pulses shorter than the original laser pulse duration being produced when the
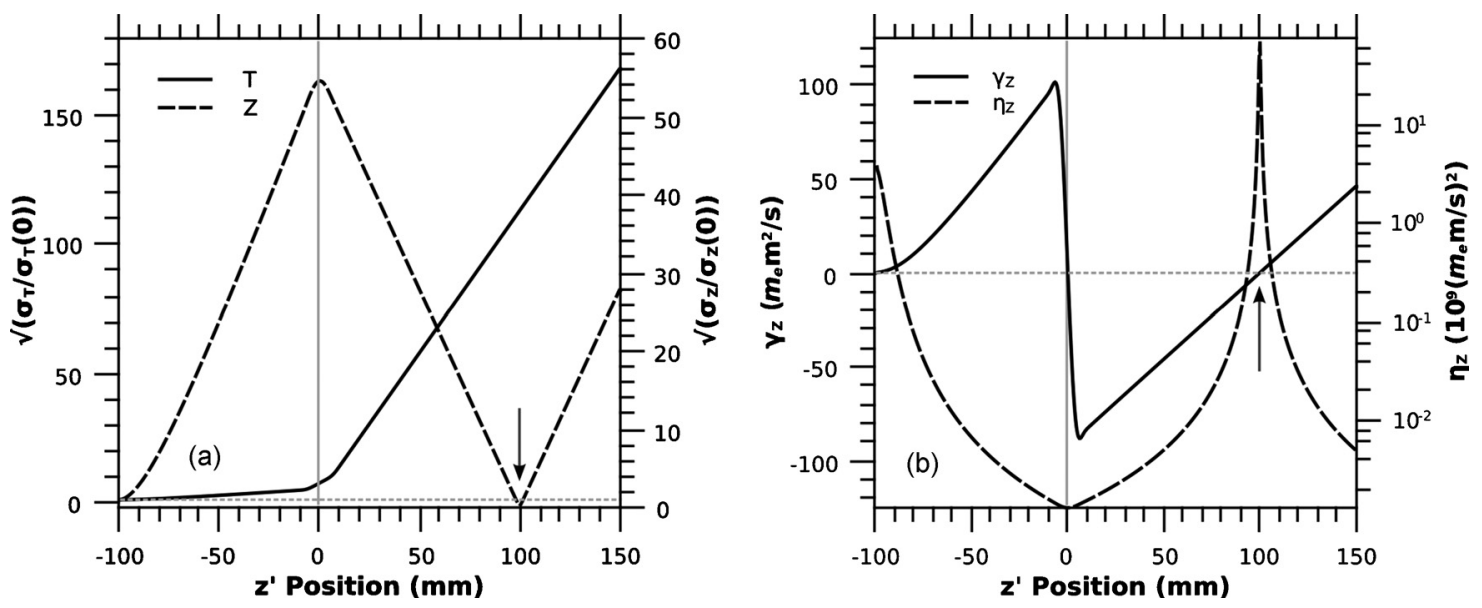

FIG. 6. Temporal focusing of an rf pulse compression cavity simulated by the AG model for $N=10^{5}$ : dependences of (a) the normalized pulse widths

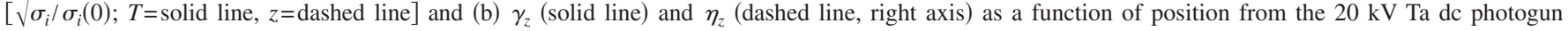
$\left(z^{\prime}=-100 \mathrm{~mm}\right)$, through the rf cavity $\left(z_{\mathrm{rf}}^{\prime}=0\right)$, and beyond the temporal focus $\left(z^{\prime}=100 \mathrm{~mm}\right)$. Initial laser pulse parameters: $w=100 \mu \mathrm{m}$ and $\tau=100 \mathrm{fs}[i . e$., $\xi(0) \approx 0.084]$. rf cavity parameters: $E_{0}=466 \mathrm{kV} / \mathrm{m}$ and $\phi=0$. The arrow indicates the position of the temporal focus. 
distance from the rf cavity to the temporal focus is less than that from the effective photocathode to the rf cavity; specifically, the ratio of the time-of-flight between the rf cavity and the temporal focus to that between the photogun and the rf cavity.

The rf electric field amplitude $E_{0}$ of about $400 \mathrm{kV} / \mathrm{m}$ required to achieve the temporal focusing $10 \mathrm{~cm}$ behind the rf cavity in the dispersive limit is also consistent with expectations. By equating the differential impulse applied by the rf cavity across the electron pulse duration to that required to compensate for dispersive pulse broadening before the rf cavity and then compress the pulse thereafter, one can show that the required rf field amplitude for a $\mathrm{TM}_{010}$ cavity with $d=\pi v_{0} / \Omega$ is given to a good approximation by

$$
E_{0} \approx V_{\mathrm{dc}}\left(\frac{1}{L_{1}}+\frac{1}{L_{2}}\right)
$$

where $L_{1}$ is the distance from the pulse source (the dc photoelectron gun) to the rf cavity (the object distance) and $L_{2}$ is the distance to the temporal pulse focus behind the rf cavity (the image distance). For the considered case, $V_{\mathrm{dc}}=20 \mathrm{kV}$ and $L_{1}=L_{2}=10 \mathrm{~cm}$, giving $E_{0} \approx 400 \mathrm{kV} / \mathrm{m}$, which is in good agreement with the optimum value of $423.5 \mathrm{kV} / \mathrm{m}$ employed for the simulation displayed in Fig. 5(b). Equation (31) also clearly indicates that the dc photoelectron gun and $\mathrm{TM}_{010}$ rf pulse compression cavity should be considered as a single system, since the temporal 'focal length' of the rf cavity, $V_{\mathrm{dc}} / E_{0}$, is dependent upon the dc photogun voltage.

The optimized rf cavity performance for the temporal compression of a pulse with the same initial photoemission conditions but with $N=10^{5}$ electrons, is shown in Fig. 6. In this case, intrapulse space-charge effects act to further broaden the initial $100 \mathrm{fs}$ pulse duration to $\sim 6 \mathrm{ps}$ after the $\sim 1$ ns time-of-flight over the $10 \mathrm{~cm}$ distance to the rf cavity. The fact that almost the same rf field strength is required to generate a temporal focus at $z^{\prime}=10 \mathrm{~cm}$ is a direct consequence of the linear dependence of the longitudinal $\mathrm{rf}$ cavity force on $z$ [Eq. (29)], which means that the required restorative force is not dependent on $\sigma_{z}$-just as the focal length of a perfect optical lens is independent of its aperture size. However, unlike in the dispersion limited case (Fig. 5), the compressed pulse duration is now a factor of $\sim 4$ less than the original $100 \mathrm{fs}$ laser pulse duration. This additional pulse compression for the employed 1-to-1 image-to-object distance (magnification) ratio is made possible by the additional pulse bandwidth (and momentum chirp) generated by the space-charge effects-in analogy to self-phase modulation (and dispersion) in fiber-grating laser pulse compression. ${ }^{34} \mathrm{In}$ this case, the $\sim 100$-fold increase in the transverse pulse size (corresponding to a $10^{4} \times$ reduced peak pulse charge density) aids the temporal compression by greatly diminishing Coulomb effects that counteract the reduction in electron pulse duration. Again, a stronger (and suitably phased) rf field will result in an even shorter compressed pulse duration as the distance to the temporal focus behind the rf cavity is reduced.

It is important to note that the example illustrated in Fig. 6 approaches the limit imposed in obtaining Eq. (28); namely that the longitudinal electron pulse length be much shorter than the axial length $d$ of the rf cavity, or $\Omega z \ll v_{0}$. For a $\sim 10$ ps electron pulse entering the $3 \mathrm{GHz}$ rf cavity we have $\Omega z / v_{0}=\Omega \tau \approx 0.19$, for which the small angle approximation $\sin \left(\Omega z / v_{0}\right)=\Omega z / v_{0}$ is $0.6 \%$ inaccurate. Again, in principle, the AG model could simulate the action of the rf cavity on longer pulse by including additional terms $O\left(z^{3}\right)$ and higher in Eq. (29) resulting from the expansion of $\sin \left(\Omega z / v_{0}\right)$, but at the expense of voiding its self-similar Gaussian approximation.

\section{COMPOUND ELEMENT SYSTEMS}

Through the use of Eq. (25) to specify the position and influence of electron optical components such as magnetic lenses and rf cavities, the modified AG model can be employed to design and assess a compound element system. The design of such a compound lens system is, of course, a cornerstone of modern electron microscope development, although space-charge effects are generally not included, since, on average, there is usually no more than one electron in the microscope column at a time. However, for a nanosecond DTEM, ${ }^{22,27}$ the microscope column must be designed to compensate for transverse $(T)$ space-charge effects, which dominate the propagation dynamics for the cigarlike electron pulses employed in such instruments (see Fig. 2). For the ultrashort (i.e., picosecond and femtosecond) electron pulse durations used in UEMs (Ref. 13) and UED experiments, ${ }^{16}$ the longitudinal $(z)$ pulse dynamics due to both dispersion and space-charge effects must also be considered (as indicated in Fig. 2).

Figure 7 shows an example of a compound element system for the delivery of an ultrashort electron pulse to the specimen area; in this case, optimized for $N=10^{6}$ - the number of electrons per pulse required for single shot diffraction measurements. ${ }^{12}$ In this simple system, an optimum rf cavity $\left(\Omega=\pi v_{0} / d\right)$ is surrounded by two magnetic lenses (positioned a realistic $5 \mathrm{~cm}$ either side of the rf cavity) that compensate for the electron beam divergence due to the nonzero $\eta_{T}(0)$ and the negative spatial lensing of the rf pulse compression cavity. The magnetic lenses are assumed to be identical (but oppositely wound to allow for the compensation of rotational effects in the magnetic focusing through the use of oppositely directed axial magnetic fields). For the transverse dimension, this arrangement of electron optical elements is similar to the Cooke triplet in optics. The displayed electron pulse dynamics are for a $\tau=100$ fs electron pulse with $w$ $=0.1 \mathrm{~mm}$ generated from a laser driven $20 \mathrm{kV}$ Ta gun $\left(v_{0}\right.$ $=8.4 \times 10^{7} \mathrm{~m} / \mathrm{s}$ ), with $\Delta E=0.5 \mathrm{eV}$ using $\hbar \omega=4.75 \mathrm{eV}$ photons, at a distance of $15 \mathrm{~cm}$ in front of the first magnetic lens. The three lensing elements are adjusted to space-time image the electron pulse $5 \mathrm{~cm}$ behind the second magnetic lens. The dependencies of the the six pulse parameters, $\sigma_{i}$, $\eta_{i}$, and $\gamma_{i}$, shown in Fig. 7 as the electron pulse propagates through the multielement system clearly illustrate the effect of the magnetic lenses and rf cavity in the transverse $(T)$ and longitudinal $(z)$ dimensions. As the image-to-object distance ratio is less than unity in this case, both the transverse and longitudinal imaged pulse dimensions are reduced relative to their initial values. 

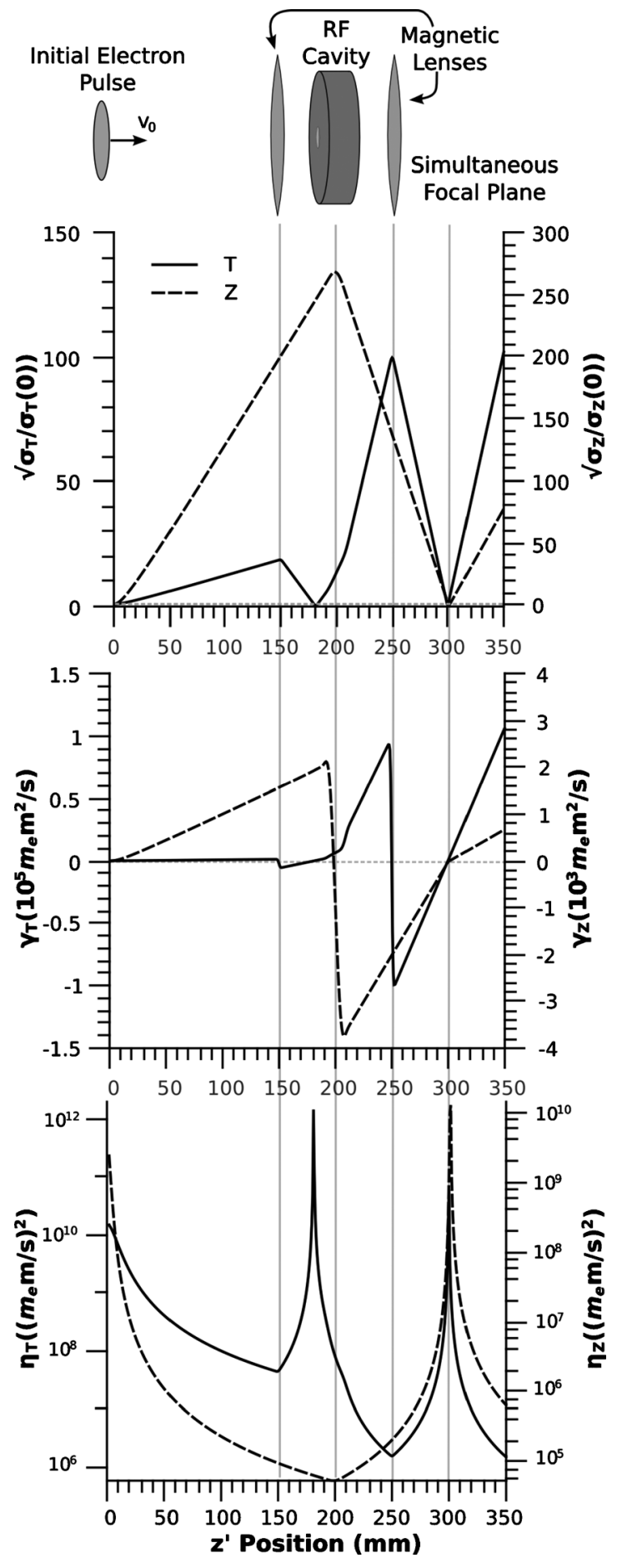

FIG. 7. A three element "optical system" for simultaneously focusing of the transverse $(T)$ and temporal $(z)$ widths of an electron pulse with $N=10^{6}$ electrons. Initial laser pulse parameters: $w=100 \mu \mathrm{m}$ and $\tau=100 \mathrm{fs}$ [i.e., $\xi(0) \approx 0.084]$.

As seen in Secs. V B and V C, with increasing pulse charge, Ne, space-charge effects in general will act to degrade the space-time imaging by increasing the threedimensional pulse size delivered to the reference plane positioned $5 \mathrm{~cm}$ behind the second magnetic lens. At $N=10^{6}$, space-charge effects moderate the image demagnification due to the $\sim 2$ object-to-image distance ratio in Fig. 7. In other words, for the displayed compound element system, which could be employed in UED experiments, ${ }^{16}$ the initial space-time resolution associated with the $\sim 0.1 \mathrm{~mm}$ spot size and $\sim 100$ fs pulse duration can be maintained (or bettered) for $N \lessgtr 10^{6}$. For a UEM, on the other hand, it may be necessary to separate longitudinally the space and time images produced by the compound element column. This is because the inclusion of the microscope's objective lens will likely require the pulse's time focus to be at the objective's back focal plane (i.e., in the specimen) while the column's spatial image may need to be located at the objective's front focal plane (i.e., entrance aperture) for efficient beam transfer with minimal spherical aberration. Fortunately, a compound component system of the type shown in Fig. 7 with a rf cavity can meet this challenge since the longitudinal positions of the two foci can be manipulated independently.

\section{SUMMARY}

We have presented an extension to the mean-field selfsimilar analytic Gaussian (AG) electron pulse propagation model of Michalik and Sipe ${ }^{1}$ that provides for the inclusion of linear external forces acting on the pulse. This extended formalism enables the model to simulate the action of electron optical elements on the electron pulse; in particular, magnetic lenses in the transverse direction and rf cavities in the longitudinal (propagation) direction. As the AG model has already been shown to accurately describe the free-space propagation dynamics of electron pulses with different shapes ${ }^{18}$ our extension then allows for a rapid first-order evaluation of electron pulse delivery systems in UED and UEM instruments. Indeed, the simulation of the compound element system shown in Fig. 7 takes only a few seconds, using Mathematica 7 with its NDSolve routine on a standard commercial PC, thus allowing computational space-time optimization for the delivery of the electron pulse in only a few minutes.

We have used the extended AG electron pulse propagation model to show that the disklike $(\xi \ll 1)$ electron pulses can be spatially focused more readily than cigarlike $(\xi \gg 1)$ pulses, and that the impulsive nature of global space-charge effects in the pulse favors the use of short focal length lenses due to the reduced time-of-flight to the focal point. For the temporal (longitudinal) direction, we show that the AG model also can be employed to simulate the compressive action of a suitably-phased $\mathrm{TM}_{010}$ rf cavity-a temporal lens; reversing the broadening effects of both dispersion and space-charge. Finally, we demonstrate that the extended AG model can be employed to aid the design of a multielement system (Fig. 7) for the independent control of the locations of the temporal and spatial electron pulse foci a specification of potential importance to UEM column design. These exemplars for the action of external forces in the transverse $(T)$ and longitudinal $(z)$ directions also provide the foundation for the inclusion of other effects such as the divergence of the dc field in the vicinity of the anode aperture in electron guns. $^{24}$

We emphasize, however, that the presented AG model extension is valid only for the action of linear forces (i.e., forces that have a linear dependence on distance in the pulse frame) on the electron pulse due to the model's self-similar 
Gaussian approximation. The inclusion of the action of nonlinear forces (e.g., due to space-charge effects, spherical magnetic lens aberrations, etc.) will require the use of more sophisticated electron pulse propagation models such as full particle tracking simulations, which should be employed to gauge the accuracy (and hence limits) of our extended AG approach at some point. Nevertheless, for many current and planned UEM and UED systems, the computational efficiency and robust nature of the extended AG model is expected to be invaluable for performance assessment, as indicated by reasonably accurate preliminary modeling of a 20 $\mathrm{kV}$ dc photoelectron gun. ${ }^{23}$ On the other hand, in some practical cases, conservation of the quantity $\sqrt{\sigma_{i} \eta_{i}}$ (the pulse emittance) which is inherent in the AG model, may not be satisfied; for example, in the presence of a beam aperture (i.e., spatial filter). Nonetheless, within the spirit of the selfsimilar AG model, an aperture may even be simulated through suitable adjustment of both $N$ and $\sigma_{T}$ at the aperture plane.

\section{ACKNOWLEDGMENTS}

This research was supported in part by the National Science Foundation under Award No. DMR-0619573 and the Department of Energy under Award No. DE-FG5206NA26213. J.A.B. acknowledges support from the Department of Education under the Graduate Assistantships in $\mathrm{Ar}$ eas of National Need (GAANN) program.

${ }^{1}$ A. M. Michalik and J. E. Sipe, J. Appl. Phys. 99, 054908 (2006); 103, 129901 (2008).

${ }^{2}$ W. E. King, G. H. Campbell, A. Frank, B. Reed, J. F. Schmerge, B. J. Siwick, B. C. Stuart, and P. M. Weber, J. Appl. Phys. 97, 111101 (2005). ${ }^{3}$ R. Srinivasan, V. Lobastov, C. Ruan, and A. Zewail, Helv. Chim. Acta 86, 1761 (2003).

${ }^{4}$ J. C. Williamson, J. Cao, H. Ihee, H. Frey, and A. H. Zewail, Nature (London) 386, 159 (1997).

${ }^{5}$ J. Cao, Z. Hao, H. Park, C. Tao, D. Kau, and L. Blaszczyk, Appl. Phys. Lett. 83, 1044 (2003).

${ }^{6}$ G. Sciaini, M. Harb, S. G. Kruglik, T. Payer, C. T. Hebeisen, F. M. zu Heringdorf, M. Yamaguchi, M. H. von Hoegen, R. Ernstorfer, and R. J. D. Miller, Nature (London) 458, 56 (2009).

${ }^{7}$ B. J. Siwick, J. R. Dwyer, R. E. Jordan, and R. J. D. Miller, Science 302, $1382(2003)$.
${ }^{8}$ A. H. Zewail, Science 242, 1645 (1988).

${ }^{9}$ O. Bostanjoglo and R. Liedtke, Phys. Status Solidi A 60, 451 (1980).

${ }^{10}$ H. Dömer and O. Bostanjoglo, Rev. Sci. Instrum. 74, 4369 (2003).

${ }^{11}$ T. LaGrange, M. R. Armstrong, K. Boyden, C. G. Brown, G. H. Campbell, J. D. Colvin, W. J. DeHope, A. M. Frank, D. J. Gibson, F. V. Hartemann, J. S. Kim, W. E. King, B. J. Pyke, B. W. Reed, M. D. Shirk, R. M. Shuttlesworth, B. C. Stuart, B. R. Torralva, and N. D. Browning, Appl. Phys. Lett. 89, 044105 (2006).

${ }^{12}$ M. R. Armstrong, K. Boyden, N. D. Browning, G. H. Campbell, J. D. Colvin, W. J. DeHope, A. M. Frank, D. J. Gibson, F. Hartemann, J. S. Kim, W. E. King, T. B. LaGrange, B. J. Pyke, B. W. Reed, R. M. Shuttlesworth, B. C. Stuart, and B. R. Torralva, Ultramicroscopy 107, 356 (2007).

${ }^{13}$ H. S. Park, O. Kwon, J. S. Baskin, B. Barwick, and A. H. Zewail, Nano Lett. 9, 3954 (2009).

${ }^{14}$ M. R. Armstrong, B. W. Reed, B. R. Torralva, and N. D. Browning, Appl. Phys. Lett. 90, 114101 (2007).

${ }^{15}$ V. A. Lobastov, R. Srinivasan, and A. H. Zewail, Proc. Natl. Acad. Sci. U.S.A. 102, 7069 (2005)

${ }^{16}$ T. van Oudheusden, E. F. de Jong, S. B. van der Geer, W. P. E. M. Op't Root, O. J. Luiten, and B. J. Siwick, J. Appl. Phys. 102, 093501 (2007).

${ }^{17}$ L. Veisz, G. Kurkin, K. Chernox, V. Tarnetsky, A. Apolonski, F. Krausz, and E. Fill, New J. Phys. 9, 451 (2007).

${ }^{18}$ A. M. Michalik and J. E. Sipe, J. Appl. Phys. 105, 084913 (2009).

${ }^{19}$ O. J. Luiten, S. B. van der Geer, M. J. de Loos, F. B. Kiewiet, and M. J. van der Wiel, Phys. Rev. Lett. 93, 094802 (2004).

${ }^{20}$ B. J. Siwick, J. R. Dwyer, R. E. Jordan, and R. J. D. Miller, J. Appl. Phys. 92, 1643 (2002)

${ }^{21}$ K. L. Jensen, P. G. O'Shea, D. W. Feldman, and N. A. Moody, Appl. Phys. Lett. 89, 224103 (2006).

${ }^{22}$ B. Reed, M. Armstrong, N. Browning, G. Campbell, J. Evans, T. LaGrange, and D. Masiel, Microsc. Microanal. 15, 272 (2009).

${ }^{23}$ J. A. Berger, J. T. Hogan, M. J. Greco, W. A. Schroeder, A. W. Nicholls, and N. D. Browning, Microsc. Microanal. 15, 298 (2009).

${ }^{24}$ K. Togawa, T. Shintake, T. Inagaki, K. Onoe, T. Tanaka, H. Baba, and H. Matsumoto, Phys. Rev. ST Accel. Beams 10, 020703 (2007).

${ }^{25}$ Y. Li and J. W. Lewellen, Phys. Rev. Lett. 100, 074801 (2008).

${ }^{26}$ B. W. Reed, J. Appl. Phys. 100, 034916 (2006).

${ }^{27}$ T. LaGrange, G. H. Campbell, B. Reed, M. Taheri, J. B. Pesavento, J. S. Kim, and N. D. Browning, Ultramicroscopy 108, 1441 (2008).

${ }^{28} \mathrm{D}$. B. Montgomery and J. Terrell, "Some useful information for the design of air-core solenoids" National Magnet Laboratory Technical Report AFOSR-1525, 1961 (unpublished).

${ }^{29}$ A. B. El-Kareh and J. C. J. El-Kareh, Electron Beams, Lenses, and Optics (Academic, New York, 1970), Vol. 1.

${ }^{30}$ E. Fill, L. Veisz, A. Apolonski, and F. Krausz, New J. Phys. 8, 272 (2006).

${ }^{31}$ S. Humphries, Principles of Charged Particle Acceleration (Wiley, New York, 1986).

${ }^{32}$ K.-J. Kim, Nucl. Instrum. Methods Phys. Res. A 275, 201 (1989).

${ }^{33}$ K. T. McDonald, IEEE Trans. Electron Devices 35, 2052 (1988).

${ }^{34}$ D. Strickland and G. Mourou, Opt. Commun. 56, 219 (1985). 